\title{
Physical Properties of Carboxymethyl Cellulose Reinforced- Sucrose Plasticised Thermoplastic Mango Starch Biofilms
}

\author{
Ernest C. Agwamba ${ }^{1,3, *}$, Lawal G. Hassan ${ }^{1}$, Abdullahi. M. Sokoto ${ }^{1}$, Mohammed Achor ${ }^{2}$, and Sirajo A. \\ Zauro $^{1}$ \\ ${ }^{1}$ Department of Pure and Applied Chemistry, Faculty of Sciences, Usmanu Danfodiyo University Sokoto \\ Sokoto, Nigeria \\ ${ }^{2}$ Department of Pharmaceutics, Faculty of Pharmaceutical Sciences, Usmanu Danfodiyo University Sokoto \\ Sokoto, Nigeria \\ ${ }^{3}$ Department of Chemical Sciences, Faculty of Sciences, Clifford University, Owerrinta \\ Abia State, Nigeria \\ ${ }^{*}$ Corresponding author's email: agwambae [AT] clifforduni.edu.ng
}

\begin{abstract}
Attempt to save the planet from numerous environmental challenges has been an on-going activity for many decades, through the use of sustainable materials and processes. These have necessitated researches in materials science driven by sustainable chemical approach to derive sustainable materials that do not depend on fossil resources for industrial feedstock, and these materials do not have a negative outcome on the environment. This study investigated the physical and intermolecular interaction of Mango starch derived bioplastic plasticised with sucrose, and reinforced with carboxymethyl cellulose (CMC). Water uptake (WU) were observed to decrease significantly as the molar concentration of $\mathrm{HCl}$ or sucrose increases independently, and increase in WU was observation when CMC was increased $(p \leq 0.05)$. Increasing the molar concentration of $\mathrm{HCl}$ or the percentage amount of sucrose as plasticiser has no effect on the oil-uptake $(\mathrm{OU})$, while increasing the percentage of CMC resulted to a decrease, which shows no effect as the CMC amount increases ( $p>0.05$ ). Vapour absorption index (VAI) showed a similar trend to WU but significantly, higher outcomes were observed ( $p \leq 0.05$ ). The FTIR results also indicate that a physical interaction has occurred between the blends increase in sucrose showed a change in the FTIR peaks especially in the broader peaks observed in the $O-H$ regions of $3500-3200 \mathrm{~cm}^{-1}$ compared to unplasticized native mango starch.
\end{abstract}

Keywords--- Thermoplastic Mango starch; Sucrose plasticiser; water uptake; oil uptake, vapour absorption index

\section{INTRODUCTION}

The continuous disadvantage of petro-plastic utilization has been a bane of debates and concern to the global government, environmental activist, and scientific communities. Renewable plastic represents a significant area of interest that holds the sustainable solution to remedy the negative impact experienced in the environment due petro-plastic use.

Remarkable fund and grants has been invested by Environmental influencers, activists, non-government organizations, researchers and industrial scholars alike to search for solutions or replacement to myriads of problems associated with conventional plastics which usually is due to its non-biodegradability character after used and disposed they persist in the environment for extended length of time running in hundreds of years [1].

There is a growing awareness among the public and populace about the dangers and consequences associated with the use and improper disposal of non-biodegradable single-use-plastics on the environment.

Plastice [2], states that due to the exceptional growth of the production and the use of polymers, consideration about the consequences of plastic products use, and the waste management of these products when they become waste is a current and pressing issue.

All effort towards recycling has not yield any tangible results as reported by Fabunmi et al., [3], as the preference of recycling is becoming highly unfeasible with the production of complex multi-phased Products, and process of recycling and incineration is thermal-degradation process that requires high amount of energy, and releases harmful greenhouse gas that results to air pollution in the environment.

Attention has been drawn to carnage taking place in the marine environment due to the pollution caused by disposal of single-use-petro-plastics made from polymers in the ocean in recent time. Apart from other global challenge like global warming, the greenhouse gas effect, plastic has contributed largely to increase in the mortality rate of marine life, either by ingestion or entanglement [4].

Intact plastic that are not consumed by large marine animals mistaking for fish, undergoes biodegradation and fragmentation into smaller bit size to form microplastics due to microbial activities, mechanical abrasion, thermo-oxidation, and UV-radiation. These factors cause embrittlement and cracking of the plastic into microplastics [5]. 
These microplastics resemble plankton, food for ocean bottom feeders and small fish, who are in turn consumed by larger fish, and these could end up on our table as a source of protein, and oil. This could be the cause of the recent rise in the case of cancer and other non-prevalent diseases that are now of public concern. [6].

Although microbial produced polyhydroxyalkanoates (PHAs) offers a promising biodegradable polymer that may alleviate some of the environmental burdens of petro-plastic but the needs for carbon substrates and energy for bioreactor operations are the major conditions contributing to the high production costs and environmental impact of PHAs [7].

Proteins, lipids, triglycerides, gluten, citric acid and polysaccharides have been investigated as biomaterial sources by numerous researchers in different regions and time for bioplastic production [8].

Among all the possible green-alternatives, polysaccharides are the most affordable, and versatile, and scientific research outcomes and from economic perspective has shown it to be the most desirable source of raw materials that can be converted via physical, chemical, or biological modifications to sustainable end-products that have found application in different fields [9], and provides an escape from the over-dependence on fossil fuel as raw materials.

The importance of materials that are more environmentally friendly has been an emphasy recently by environmentalists' and academic researchers' concern which have come to stay, since the usual draws-backs associated with conventional petro-plastics will not be encountered. Concluded and ongoing scientific study has continued to give positive feedback about polysaccharide-based bioplastic with outstanding outcomes that have significantly continued to show market potential to replace petro-plastics [10].

Thermoplastic starch currently represents the most important and widely used bioplastic made by varying the amounts of additives and plasticiser tailored to specific needs in the characteristic of the biomaterial.

The introduction of modifiers such as plasticizer (urea, ethanolamine, Formamide, Sorbitol and glycerol) to the starch polysaccharide chain, is it improves the flexibility [11]. Most Plasticizer contain hydroxyl groups that allow compatibility with starch granules and then plasticizer starch by breaking the internal hydrogen bonding between the glucose rings to improve the starch. This resulted to a biopolymer that has a continuous phase and flows when the glass transition temperature is reached. Abbot et al., [12], was able to synthesized thermoplastic starch with Ammonium salt and urea as plasticiser, and obtained a result that shows promising mechanical, and physical properties like transparency under hot-compression better than conventional petro-plastics like High density polyethylene (HDPE).

The use of monosaccharide and oligosaccharides as plasticiser as a study has been reported, hence the need for this study to investigate the use of sucrose as the plasticiser.

The main aim of this study is the investigate the influence of variable amount of sucrose as plasticiser, carboxymethyl cellulose (CMC) and molar concentration of $\mathrm{HCl}$ on the physical properties of thermoplastic mango starch and also the nature of intermolecular interaction between the components of the thermoplastic will be investigated with the help of FTIR device/analysis.

\subsection{Materials}

\section{MATERIALS AND METHODS}

Pristine mango starch used for this study was obtained from the previous published investigation [13]. All other chemicals used were of laboratory reagent (LR).

\subsection{Preparation of bioplastic derivative film}

With some modifications, method described by Wissinger et al., [14] was adopted. To a $50 \mathrm{~cm}^{3}$ beaker, $13.00 \mathrm{~cm}^{3}$ of distilled water was added, followed by $1.25 \mathrm{~g}$ starch powder, $2.0 \mathrm{~cm}^{3} \mathrm{HCl}$ acid $(0.00,0.10,0.15$, or $0.20 \mathrm{M}$.), and sucrose $(0,80,180 \% \mathrm{w} / \mathrm{w})$ was added, carboxymethyl cellulose $(0,30$, or $70 \% \mathrm{w} / \mathrm{w})$. The mixtures were placed on a magnetic stirrer, heated, and stirred gently on a hot plate. The mixture started out in white colour and changes to transparent or translucent and thickened. Once the initial white colour of the starch was completely changed and the mixture thickened, $2.0 \mathrm{~cm}^{3}$ of $\mathrm{NaOH}_{(\mathrm{aq})}$ was added to neutralise the acid (the same concentration with $\mathrm{HCl}_{(\mathrm{aq})}$ acid) and removed from the heat. The hot mixtures were gently cast into a labelled petri-dish, and dried in the oven for 6 hours at $70{ }^{\circ} \mathrm{C}$.

\begin{tabular}{clll}
\multicolumn{4}{c}{ Table 1.1: Shows the Un-coded parameter for Design of Experiment } \\
$\begin{array}{llll}\text { Sample } \\
\text { code }\end{array}$ & Sucrose $(\%)$ & CMC (\%) & HCl (M) \\
\hline SB1 & 0.00 & 30 & 0.10 \\
SB2 & 180 & 30 & 0.10 \\
SB3 & 80 & 0.00 & 0.10 \\
SB4 & 80 & 70 & 0.10 \\
SB5 & 80 & 50 & 0.10 \\
SB6 & 80 & 30 & 0.00 \\
SB7 & 80 & 30 & 0.20 \\
SB8 & 80 & 30 & 0.10 \\
SB9 & 120 & 30 & 0.10 \\
SB10 & 80 & 30 & 0.15 \\
\hline SB:
\end{tabular}

SB: sucrose plasticised bioplastic. CMC: carboxymethyl cellulose 


\subsection{Physicochemical properties}

a. Water Uptake (WU)

Water uptake was investigated by cutting samples to approximately $2 \times 2 \mathrm{~cm}$ and then weighed. The cut samples were placed into a container filled with distilled water for 24 hours. After immersion in water, samples were removed from the water and allowed to dry on a laminated paper for about 10 minutes and weighed to measure the wet weight. Water uptake was calculated as following [15].

Water uptake $=($ wet weight - dry weight $) /$ wet weight $\times 100 \%$

\section{b. $\quad$ Oil Uptake (OU)}

Oil uptake was calculated using the method described for water uptake but with ground/peanut oil as the immersion medium. The samples were washed with pure ethanol to remove excess oil and allowed to dry on a filter paper for about 10 minutes before weighing.

\section{c. $\quad$ Vapour absorption index (VAI)}

Each bioplastic specimen was accurately weighed and placed in a dissector in an oven set at $30{ }^{\circ} \mathrm{C}$. The relative humidity was controlled by using a supersaturated salt solution of $\mathrm{LiCl}, \mathrm{MgCl}_{2}, \mathrm{NaBr}, \mathrm{NaCl}$ and $\mathrm{BaCl}_{2}$,of an equal amount (10 g each in $20 \mathrm{~cm}^{3}$ of distilled water) where the water activities of the oversaturated solution differ depending on the relative humidity of the salt. Weight of biofilms was ascertained after four weeks (final weight). The weight gained and the percentage of water absorbed were considered.

Vapour absorption $=\underline{(\text { Final weight }- \text { Initial weight })} \times 100 \%$

$$
\text { Final weight }
$$

\subsection{Fourier Transformed Infrared Spectroscopy (FT-IR) analysis}

Samples were dried and IR spectra were recorded with an Agilent-FTIR spectrometer, at $27{ }^{\circ} \mathrm{C}$ from 650 to $4000 \mathrm{~cm}^{-1}$ range, background scanned at 64, sample scanned at 32, resolution was 4 and fitted with a compatible PC running the recommended operating system.

\section{RESULTS AND DISCUSSIONS}

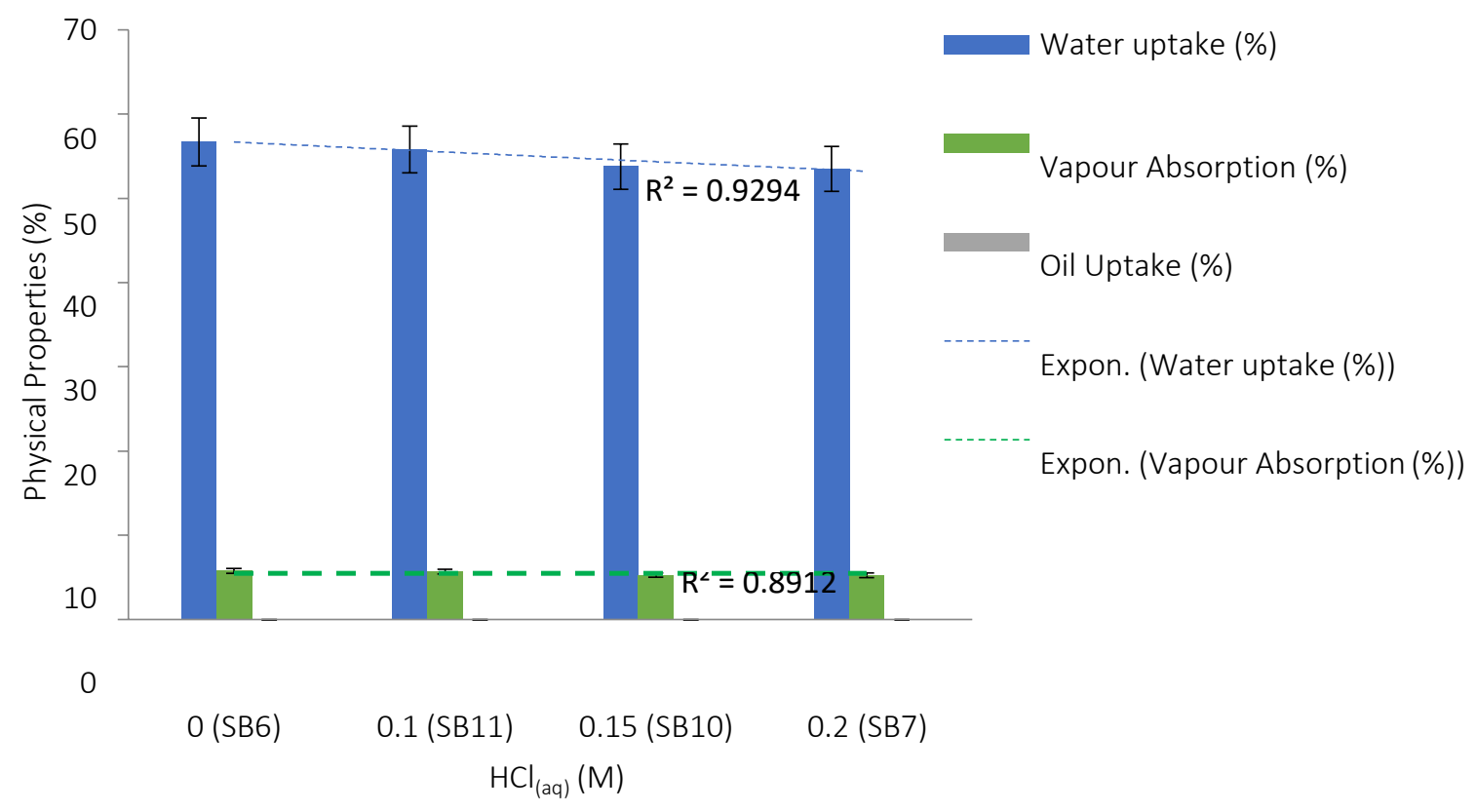

Figure 1: Effect of $\mathrm{HCl}$ concentration on Water Uptake, Vapour absorption, and Oil uptake of 80\% sucrose plasticised thermoplastic Mango Starch derivatives

Figure 1 shows an insignificant decrease in water uptake capacity as the concentration of $\mathrm{HCl}$ increases 0.00 to $0.20 \mathrm{M}$ (p $\leq 0.05)$. The vapour absorption index also indicated a declining trend that is insignificant as the molar concentration of $\mathrm{HCl}$ is elevated $(\mathrm{p}>0.05)$ just as observed for water uptake. This effect may be attributed to the increasing introduction of $\mathrm{HCl}$ molar concentration increases protonation within the thermoplastic film blend, which in turn promotes a higher intermolecular interaction between the components of the thermoplastic biofilms.

From the Figure, it revealed that bioplastic derivatives plasticised with sucrose have no oil uptake as the molar concentration of $\mathrm{HCl}$ increases $(\mathrm{p}>0.05)$. All biofilms derivatives showed excellent oil barrier, and do not absorb oil 
when immersed for 24 hours. Increasing the concentration of $\mathrm{HCl}$ inhibits the capacity of the material to absorb more oil. This could be due to the strong intermolecular force of attraction within the bioplastic-derivatives, or the bioplastic been a lipophobic material creating no intermolecular interaction between them. CMC is a lipophobic biopolymer whose presence in the thermoplastic biofilms acts as an excellent oil repellent and do not allow any form intermolecular interaction between the components of the thermoplastic biofilms even as the molar concentration of acid was increased.

This implies that the molar concentration of $\mathrm{HCl}$ is not an important factor that affects the physical characteristic of thermoplastic mango starch except maybe at higher molar concentration a significant effect could be observed.

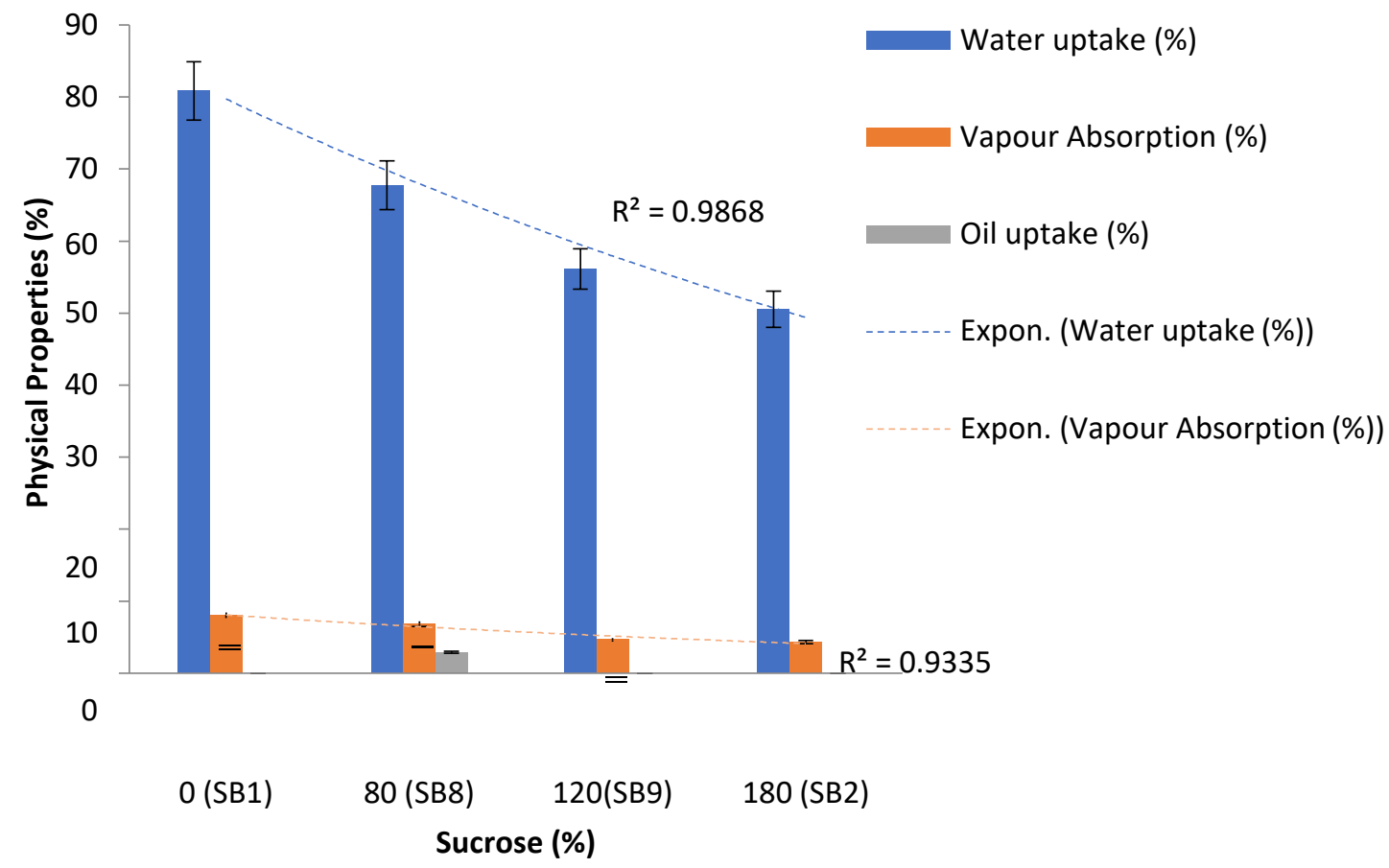

Figure 2: Effect of Sucrose on Water uptake, Vapour absorption, oil uptake of thermoplastic mango starch-30 \% CMC Reinforced derivatives

Figure 2, showed that increase for sucrose from $0 \%-180 \%$, there was a significant decrease in water uptake capacity and vapour absorption index of thermoplastic biofilms $(p \leq 0.05)$. Increasing the plasticizer enhances the water barrier of bioplastic derivatives plasticized with sucrose. Increasing the amount of sucrose result to increase in the intermolecular force of attraction between the anhydrous glucose unit in the starch biopolymer chain and the sucrose and gave no room for water molecules to percolate the matrixes of the biofilms. Therefore, increase for sucrose as a plasticizer in bioplastic reinforced with CMC reduces the permeability to water and enhances the barrier. Study by Maulida et al., [16] shows a similar trend with this work that increasing the percentage amount of Sorbitol as plasticizer results to decrease in water uptake.

Figure 2 also revealed that increase for sucrose, as a plasticiser has no effect on the oil uptake capacity of the bioplastic derivative, meaning that there is no significant relationship between the oil uptake capacity and percentage amount of sucrose plasticizer $(\mathrm{p}>0.05)$. The addition of sucrose makes the bioplastic to be lipophobic, and decreases the oil penetration capacity into the matrix of the bioplastics derivatives. The fact that oil floats in water could be same reason why oil could not penetrate these bioplastic matrices under investigation i.e. intermolecular attraction plays vital role in this observed behaviour, since oil only dissolves solute of low polarity of which the bioplastic derivatives are not but instead are polar in addition to hydrogen bonding. 


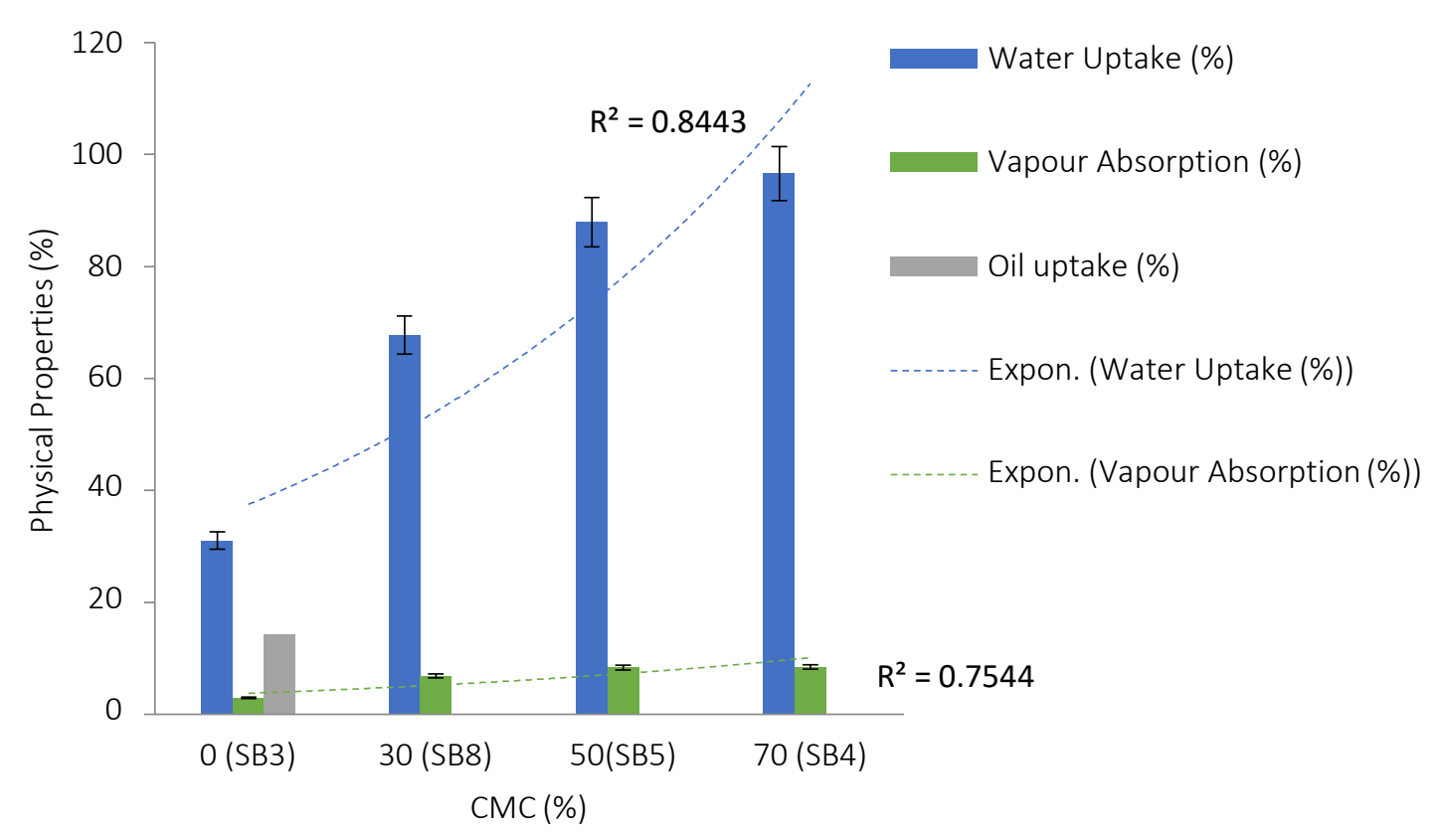

Figure 3: Effect of CMC on Water uptake Water Uptake, Vapour absorption, and Oil uptake of $80 \%$ sucrose plasticised thermoplastic Mango Starch derivatives

Figure 3 showed that as the percentage amount of CMC was increased from $0 \%-70 \%$, the water uptake increases significantly $(\mathrm{p} \leq 0.05)$ but increases insignificantly in vapour absorption index $(\mathrm{p}>0.05)$. CMC is an excellent water absorber, and has the ability to reduce the water barrier of the polymer when used as biocomposite. The result SB3 at $70 \%$ CMC showed a significantly high water uptake after 24 hours of $83.9 \%$. This result suggested that increasing the amount of hydrophilic polymer result to increase in water uptake, and has the opposite effect when hydrophobic polymer is used as additive or blended with thermoplastic starch.

Figure 3 also revealed that increase for \% CMC significantly decreases the oil uptake capacity $(\mathrm{p} \leq 0.05)$. It was indicated from Figure 3 that increasing the \% CMC results to a remarkable decrease in oil uptake capacity. SB3 was formulated with zero CMC showed significant oil uptake but at 30\%-70\% CMC no oil uptake was observed. Increase in CMC only result in a bioplastic material with increased barrier for oil, perhaps the bioplastic has strong and increased intermolecular interactions and repel the oil molecules due to polarity disparities. This indicated that CMC is highly lipophobic, and increasing the amount insignificantly affects the oil uptake capacity. This is a very important property as the thermoplastic could be used in oily food packaging.

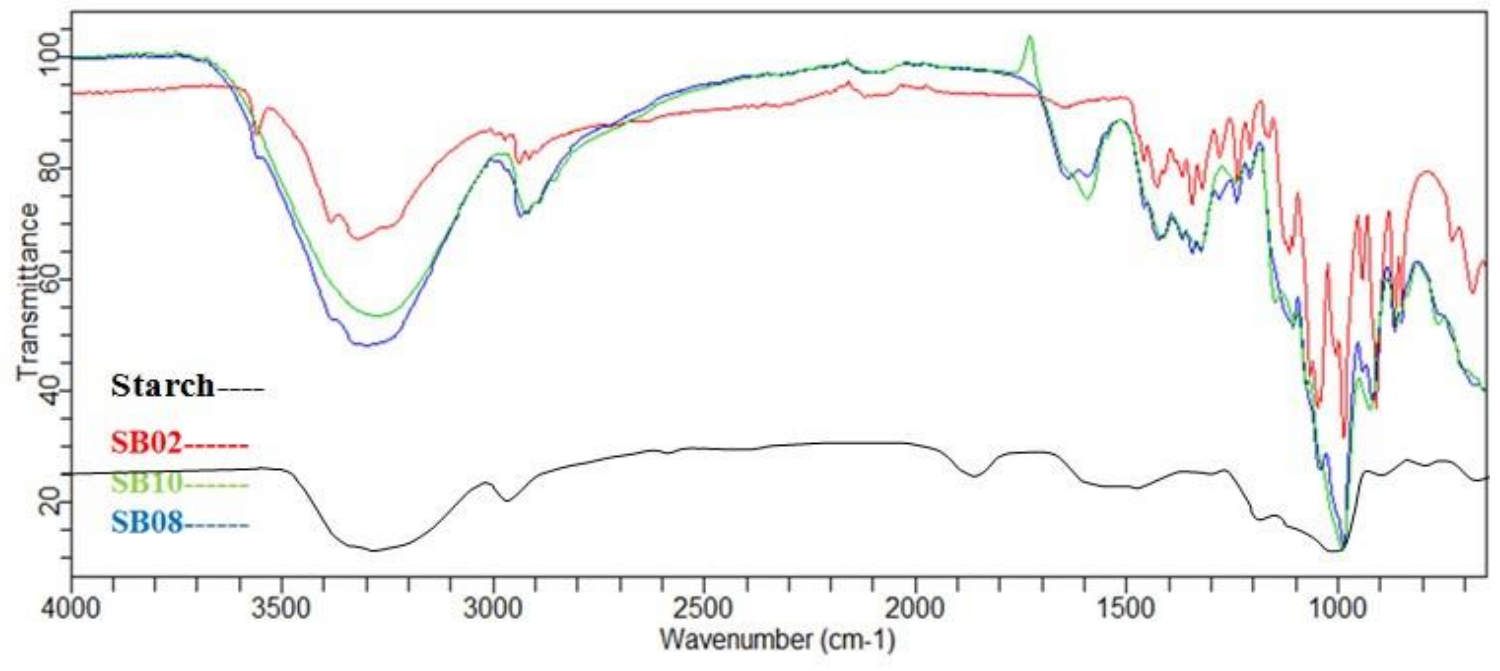

Figure 4: Overlaid FTIR Spectra of Starch from Mango, and bioplastic derivatives plasticised with variation of Sucrose $(\%)$ 


\section{FTIR Spectrum Determination for Bioplastic composite}

From Figure 4, the special pattern of typical native starch, in the region of 970 and $1200 \mathrm{~cm}^{-1}$ is preserved in CMC which is the fingerprint region of all the spectra. The CMC carboxylate (-COO-) gives the strong bands at about 1646.30, 1422.55 and $1360 \mathrm{~cm}^{-1}$. The broad band between 3600 and $3000 \mathrm{~cm}^{-1}$ is attributed to $\mathrm{O}-\mathrm{H}$ stretching and hydrogen bonding interaction involving the hydroxyl groups on the starch, sucrose and CMC molecules, and at $2929.97 \mathrm{~cm}^{-1}$ to $\mathrm{C}-\mathrm{H}$ symmetrical stretching vibrations; by carboxymethylation the intensity of both bands decreases. SB2 with $180 \%$ sucrose show more rough-multiple peaks, between $1500 \mathrm{~cm}^{-1}$ to $700 \mathrm{~cm}^{-1}$, and the roughness of the peaks reduces as the amount of sucrose decreases from $80 \%$ in SB8 to $0 \%$ in pristine mango starch.

\section{CONCLUSION}

This study indicates that sucrose plasticised thermoplastic mango starch biofilm reinforced with CMC, has good physical properties that could enable its suitability in technical applications and as single-used disposable plastic only with little modification. Sucrose is a suitable and excellent plasticiser for the production of bioplastic with enhanced water and vapour barrier. This sucrose-plasticised thermoplastic mango starch could be used in the production of single-use-plastics as disposable eating utensil due to their enhanced oil barrier for oily food. Future studies of this mango starch based bioplastic will be cantered on their biodegradability in water and soil to ascertain their duration in the environment due to pollution issues.

\section{ACKNOWLEDGEMENTS}

The authors acknowledge the assistance of Mr. Ezeayi Ajike of the Department of Physical Sciences, Faculty of Sciences, Clifford University Owerrinta (Ihie Campus), Abia State Nigeria for his assistance during the course of this study.

\section{REFERENCES}

[1] S. Pradhan, "Optimization and characterization of Bioplastic Produced by Bacillus Cereus SE1," Mational Institute of Technology, Rourkela, 2014.

[2] Plastice, "Innovative Value Chain Development For Sustainable Plastics in Central Europe," Central Europe Program and Co-financed by the European Regional Development, 2012. [Online]. Available: www.plastice.org. [Accessed 7 August 2018].

[3] O. O. Fabunmi, I. G. Tabil-Jr, S. Panigrahi and P. R. Chang, "Developing Biodegradable Plastics from Starch," in ASABE Section Meeting Presentation:, 2007.

[4] UNEP, “Biodegradable Plastics \& Marine. Misconceptions, Concerns and Impacts on Marine Environments.," in United Nations Enviroment Programme(UNEP), Nairo, 2012.

[5] A. Andrady, "Microplastics in the Marine Environment," Marine Pollution Bulletin, vol. 62, no. 8, pp. 1596-1605., 2011.

[6] GESAMP, "Sources, Fate and Effects of Microplastics in the Marine Environment - a Global Assessment. GESAMP Reports and Studies Series (2015).," GESAMP (IMO/FAO/UNESCO-IOC/UNIDO/WMO/IAEA/UN/UNEP/UNDP Joint Group of Experts on the Scientific Aspects of Marine Environmental Protection), 2016.

[7] W. Blunt, D. B. Levin and N. Cicek, “ Bioreactor Operating Strategies for Improved Polyhydroxyalkanoate (PHA) Productivity.," Polymers, vol. 10, no. 1197, pp. 1-29., 2018.

[8] M. Felix, V. Perez-Puyana, A. Romero and A. Guerrero, "Production and Characterization of Bioplastics Obtained by Injection Moulding of Various Protein Systems," Journal Polymer Environment, vol. 4, no. 8, p. 472 - 476, 2016.

[9] R. Dassanayake, S. Acharya and N. Abidi, "Biopolymer-Based Materials from Polysaccharides: Properties, Processing, Characterization and Sorption Applications," in Advanced Sorption Process Applications, intechopen, 2018.

[10] M. Lackner, “Biobased Plastics as Renewable and/or Biodegradable Alternatives to Petro-plastics,” in Kirk-Othmer Encyclopaedia of Chemical Technology, US, Wiley, 2015.

[11] Z. A. Tariq, "Physical and Chemical Investigations of Starch Based Bio- Plastics. PhD Thesis," University of Leicester, Leicester City, 2015.

[12] A. Abbott, A. D. Ballantyne and J. P. Conde, "Salt Modified Starch: Sustainable, Recyclable Plastics," Green Chemistry, vol. 14, pp. 1302-1307, 2012.

[13] E. Agwamba, L. Hassan, M. Achor and A. M. Sokoto, "Taguchi Optimization of Carboxymethylation Process and Effect Reaction Efficiency on Swelling Capacity,” Asian Journal of Applied Sciences, vol. 7, no. 5, p. $528536,2019$.

[14] J. Wissinger, P. Harris, A. Johnson, C. Ahrenstorff and L. Seifert, "Make it and Break it: Bioplastic from Plant Starch. University of Minnesota Centre for Sustainable Polymers. : 1-9.," University of Minnesota Centre for Sustainable Polymers (A NSF Centre for Chemical Innovation), Minnesota, 2016. 
[15] A. Jones, M. A. Zeller and S. Sharma, “Thermal, Mechanical, and Moisture Absorption Properties of Egg White Protein Bioplastics with Natural Rubber and Glycerol," Progress in Biomaterials, vol. 2, no. 12, pp. 1-13, 2013.

[16] M. Maulida, M. Siagian and P. Tarigan, "Production of Starch Based Bioplastic from Cassava Peel Reinforced with Microcrystalline Cellulose Avicel PH101 using Sorbitol as Plasticiser,” Journal of Physics: Conference Series, vol. 710, pp. 1-7, 2016. 